\title{
La Revista Médica de Chile en el año 2012
}

\author{
HUMBERTO REYES B. ${ }^{1}$, ATILIO BUSTOS-GONZÁLEZ ${ }^{2,3, \mathrm{a}}$, \\ MAX ANDRESEN H. ${ }^{4}$, JOAQUÍN PALMA H. ${ }^{4}$
}

\section{Revista Médica de Chile in the year 2012}

During the year 2012, 539 manuscripts were submitted to this journal, following an increasing trend in the recent decade. Rejection rate was 33\%. This higher number of submissions demanded a larger number and wider scope of external reviews, retarding the editorial process. The mean time lapse from reception to acceptance (or rejection) was 6.3 months (range 2-14) and from acceptance to publication 5.3 months (range 3-7). Research articles were 43.9\% of published manuscripts and the remaining articles were Reviews, Special Articles, Case Reports, articles on Medical Ethics, Medical Education, Evidence Based Medicine, Public Health, History of Medicine, Letters to the Editor and others. Thirty seven published manuscripts (14.6\%) came from foreign countries and 9 of them were published with full text in English. The 2012 Impact Factor was 0.360, showing little variation from previous years, locating the journal in the upper part of quartil 4 in the ISI-JCR category "Medicine, General and Internal", while the SCImago Journal \& Country Rank locates the Revista in quartil 2 of its category "Medicine (miscellaneous)". In contrast with the low citation rate, the number of visits to the open access electronic version in www.scielo. cl averages over 3 million visits per year, illustrating that the interest among readers outnumbers the country's expectable readership. Only 22\% of articles declared to have received financial help from entities external to the institution where the work was performed, coming mainly from Chilean Governmental competitive funds. The aim of Revista Médica de Chile is to provide readers and authors a valuable source of information about current relevant topics in general and internal medicine, progress in related specialties and updates in basic sciences, rendering them available to Health professionals in Chile and worldwide, following international standards of ethical and scientific quality in medical publications.

(Rev Med Chile 2013; 141: 1049-1056).

Key words: Journalism, medical; Periodicals; Scientometrics; Writing.

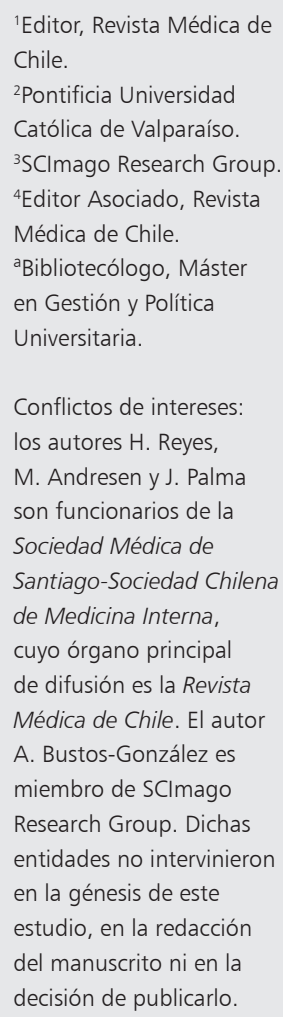

E n julio de 2012 la Revista Médica de Chile cumplió ciento cuarenta años de publicación continua. Ocupa el lugar número veinte de antigüedad entre las veinticuatro revistas médicas fundadas en el Siglo XIX que se mantienen activas, en una nómina encabezada por el New England Journal of Medicine (sucesora de una revista fundada en 1812). Solamente dos de estas veinticuatro revistas se publican en idioma español: Gaceta Médica de México (fundada en 1864) y Revista Médica de Chile (fundada en 1872).

La Sociedad Médica de Santiago-Sociedad Chilena de Medicina Interna, entidad que creó la
Revista como su órgano oficial, celebró ese aniversario el 14 de agosto de 2012 en un acto al que fueron invitados los directores de la Sociedad, sus ex presidentes, decanos de Facultades de Medicina, autoridades del Colegio Médico de Chile y del Ministerio de Salud, y otras personalidades. En dicho acto, el Editor Jefe resumió aspectos históricos relevantes en la evolución de la Revista e informó sobre su marcha, con datos numéricos hasta 2011.

En el presente artículo exponemos los principales datos de ese informe, actualizados al año 2012, comparándolos con lo vivido en años previos y con algunos estudios bibliométricos relevantes. 
Cantidad de artículos publicados en la Revista

La cantidad de artículos que se publican en la Revista depende principalmente del espacio disponible, que corresponde a un promedio de 136 páginas por ejemplar. Una causa para este límite es el costo de su impresión, que asume por completo la Sociedad Médica de Santiago recurriendo a las cuotas societarias, la recaudación por avisos comerciales y otros ingresos de la institución.

A diferencia de lo que ocurría en décadas previas, el aporte de manuscritos supera hoy ampliamente el espacio disponible, lo cual es uno de los factores que determinan la demora en publicar los que son aceptados. Para dar cabida a un número mayor de manuscritos y reducir la demora de publicación se han establecido límites a la extensión de los manuscritos, según su naturaleza y la Sección a que estén destinados, lo cual se explicita en las Instrucciones a los Autores.

\section{El flujo de manuscritos durante el año 2012 y el tiempo que toma el proceso editorial}

La Tabla 1 muestra el crecimiento progresivo que ha tenido el número de manuscritos recibidos anualmente, entre los años 2000 y 2012, con tendencia a estabilizarse en los tres últimos años. También ha aumentado la proporción de rechazos, cuya cifra actual (33\%) es inferior a la que exhibe la mayoría de las revistas médicas con alto prestigio internacional, donde varía entre 60 y $90 \%$. Nuestra tasa de rechazos se relaciona con la misión educacional adoptada por los editores, que nos impulsa a dar una oportunidad a los autores para mejorar y reenviar manuscritos cuya versión original presentaba debilidades que probablemente habrían motivado su rechazo definitivo en otras revistas.

El crecimiento en la oferta de manuscritos que tratan temas de interés médico general y tienen una presentación formal adecuada, aumenta el número de manuscritos que deben ser sometidos al proceso editorial completo, hasta su envío a los Impresores. Ello indujo a la Sociedad Médica de Santiago a cobrar por la recepción de manuscritos, dado que su manejo administrativo genera un costo superior a lo histórico; esta medida rige desde julio de 2012.

El proceso editorial comprende tres etapas cronológicas cruciales: la fecha de recepción del manuscrito, la de su aceptación y la de su publicación en la versión impresa de la Revista ${ }^{1-3}$. En el año 2012, el tiempo promedio desde la recepción de un Artículo de Investigación hasta su aceptación (o el rechazo definitivo) fue 6,3 meses (rango de 2 a 14 meses). Este promedio estuvo influido negativamente por 11 de los 111 Artículos de Investigación publicados $(9,7 \%)$, los cuales demoraron 11 a 14 meses en esta etapa; ello se debió fundamentalmente al tiempo ocupado por los autores para corregir su manuscrito y reenviarlo a la revista. Una vez aceptados, el tiempo promedio que demoraron los Artículos de Investigación hasta ser publicados fue 5,3 meses (rango 3 a 7). Para los Artículos de Revisión, Casos Clínicos y los ubicados en otras Secciones, la demora del proceso editorial ha sido diferente, dependiendo de distintos factores.

$\mathrm{Al}$ aumentar el flujo de manuscritos ha sido necesario aumentar sustancialmente el número de revisores externos. Mientras en el año 2000 se consultaron 143 revisores, en el año 2012 fueron 534 personas y cada una revisó uno a tres manuscritos. Cabe señalar que un factor relevante en la demora del proceso editorial es el tiempo que toma conseguir la aceptación para revisar un manuscrito y luego recibir el informe de los revisores asignados.

Treinta y siete manuscritos publicados en el año 2012 procedían del extranjero (Tabla 2), equivalentes a $14,6 \%$ de los "artículos citables" ("citables" corresponde a una calificación que incluye solamente a los manuscritos sometidos

Tabla 1. Número de manuscritos recibidos y rechazados, anualmente, en la Revista Médica de Chile. Tendencia observada entre los años 2000 y 2012

\begin{tabular}{|ccccccccc|}
\hline Manuscritos / años & $\mathbf{2 0 0 0}$ & $\mathbf{2 0 0 3}$ & $\mathbf{2 0 0 5}$ & $\mathbf{2 0 0 8}$ & $\mathbf{2 0 0 9}$ & $\mathbf{2 0 1 0}$ & $\mathbf{2 0 1 1}$ & $\mathbf{2 0 1 2}$ \\
Recibidos $(\mathrm{n})$ & 228 & 296 & 366 & 376 & 434 & 547 & 520 & 539 \\
Rechazados $(\%)$ & 13 & 12 & 20 & 33 & 20 & 24 & 28 & 33 \\
\hline
\end{tabular}


Tabla 2. Manuscritos procedentes del extranjero, publicados en 2012 en la Revista Médica de Chile, según Secciones

\begin{tabular}{|c|c|c|c|c|c|c|}
\hline País de origen & $\begin{array}{c}\text { Artículo } \\
\text { Investigación }\end{array}$ & $\begin{array}{l}\text { Artículo } \\
\text { Revisión }\end{array}$ & $\begin{array}{l}\text { Artículo } \\
\text { Especial }\end{array}$ & $\begin{array}{l}\text { Casos } \\
\text { Clínicos }\end{array}$ & $\begin{array}{l}\text { Cartas al } \\
\text { Editor }\end{array}$ & Total \\
\hline España & 3 & 2 & & 1 & 6 & 12 \\
\hline Argentina & & & 3 & 1 & 2 & 6 \\
\hline Brasil & 3 & & & 2 & & 5 \\
\hline Perú & 1 & & & 1 & 2 & 4 \\
\hline México & 2 & & & & & 2 \\
\hline Colombia & 1 & & & & 1 & 2 \\
\hline Corea del Sur & 1 & & & & & 1 \\
\hline EE.UU. de NA & & & 1 & & & 1 \\
\hline Italia & & & & 1 & & 1 \\
\hline China & & & & & 1 & 1 \\
\hline Taiwán & & & & & 1 & 1 \\
\hline Reino Unido & & & & & 1 & 1 \\
\hline Total & 11 & 2 & 4 & 6 & 14 & 37 \\
\hline
\end{tabular}

a revisión por pares y excluye a los artículos de crónica, obituarios y otros). Nueve de ellos (3,5\%) se publicaron en idioma inglés. La mayoría de estos manuscritos provino de España, Argentina o Brasil. En comparación, en el año 2008 se publicaron 19 manuscritos procedentes del extranjero, equivalentes a $9,8 \%$ del total de manuscritos publicados ese año.

El uso del correo electrónico y del sistema OJS (“Open Journal System") en Internet ha reemplazado a la recepción de manuscritos impresos en papel. No se ha logrado aún completar el sistema OJS para aplicarlo al manejo de manuscritos por los revisores externos, pero se está trabajando para solucionarlo.

\section{Tipologías documentales publicadas durante} 2012

La Tabla 3 muestra el número de artículos publicados en cada Sección de la Revista en el año 2012. La mayor proporción fueron Artículos de Investigación. Algunos manuscritos inscritos por sus autores para la Sección Salud Pública tenían la estructura de Artículos de Investigación o de Revisión y fueron reclasificados como tales por los editores.
Tabla 3. Número de artículos publicados en la Revista Médica de Chile durante el año 2012 y su proporción según Secciones temáticas

\begin{tabular}{|lrr|}
\hline Sección & n & \% \\
\hline Editoriales & 2 & 0,8 \\
\hline Artículos de Investigación & 111 & 41,9 \\
\hline Casos Clínicos & 45 & 17,0 \\
\hline Artículos de Revisión & 24 & 9,1 \\
\hline Artículos Especiales & 12 & 4,5 \\
\hline Ética Médica & 5 & 1,9 \\
\hline Educación Médica & 5 & 1,9 \\
\hline Medicina Basada en la Evidencia & 6 & 2,3 \\
\hline Salud Pública & 1 & 0,4 \\
\hline Historia de la Medicina & 6 & 2,3 \\
\hline Documentos & 1 & 0,3 \\
\hline Cartas al Editor & 36 & 13,6 \\
\hline Crónica & 8 & 3,0 \\
\hline Reseñas Bibliográficas & 3 & 1,1 \\
\hline Índice Anual & 1 & 100 \\
\hline Total de artículos publicados & 266 & \\
\hline
\end{tabular}




\section{El posicionamiento internacional de la Revista Médica de Chile}

Desde la década de 1960, el mundo de las ciencias, agregándose posteriormente el de las humanidades, recurre a la medición de algunas características numéricas de las publicaciones para comparar su posicionamiento con el de otras revistas en su misma área temática. Este proceso ha sido liderado por el Institute of Scientific Information (ISI), actualmente Thomson Reuters, una empresa privada que publica el Journal of Citation Reports (JCR), con acceso restringido a sus suscriptores. Entre los llamados "índices bibliométricos" (o "cienciométricos") que expone el JCR, el factor de impacto de cada revista (en inglés "IF", por "Journal Impact Factor", abreviatura convencional que utilizaremos en este artículo) se ha convertido en un elemento utilizado universalmente por el mundo académico (léase "universidades") y los organismos gubernamentales (p.ej. CONICYT, en Chile) al evaluar a postulantes para adjudicarles recursos de investigación o para promoverlos en su carrera profesional. Aunque el JCR proporciona también otros índices bibliométricos, el "IF" es el indicador usado más frecuentemente ( $\mathrm{y}$ a veces el único) por las entidades que financian los proyectos de investigación y por las universidades al calificar la productividad de sus académicos en publicaciones $^{4-9}$.
En su clasificación temática "Medicine, General and Internal”, el JCR incluyó en el año 2012 a 233 revistas. La Tabla 4 compara la ubicación de algunas de ellas en esta área temática, según su "IF". La Revista Médica de Chile está ubicada en el cuartil inferior (Q4); sin embargo, su factor de impacto promedio de los últimos 5 años (" 5 -year IF”) no difiere sustancialmente del de otras revistas publicadas en español ${ }^{10}$.

En las revistas médicas, las probabilidades de originar citas bibliográficas serían más altas para los meta-análisis y las actualizaciones de temas con interés inmediato, seguidos en frecuencia por los consensos sobre diagnóstico o tratamiento y después vendrían los artículos de investigación. En cambio, los casos clínicos y artículos de otra naturaleza generan muy pocas citas bibliográficas. Basados en estas situaciones prácticas, algunos expertos aconsejan a los editores que den preferencia a publicar manuscritos con mayor probabilidad de generar citas, para aumentar el numerador de la ecuación con que se calcula el "IF" en el JCR (número de citaciones recibidas por los "artículos citables" que publicó la revista en el año en estudio) y que, simultáneamente, reduzcan el tamaño del denominador (dado por la suma de los “artículos citables" publicados por la revista en los dos años previos al que se está evaluando).

En décadas recientes han aparecido entidades privadas que emplean otros criterios para juzgar la

Tabla 4. Ubicación de algunas revistas médicas en la categoría "Medicine, General and Internal", ordenadas según su Factor de Impacto ("IF") en el año 2012*

\begin{tabular}{|c|c|c|c|c|}
\hline $\begin{array}{c}\text { n orden } \\
\text { según "IF" }\end{array}$ & Título Revista & "IF" & "5-year IF" & "Q" \\
\hline 1 & N Engl J Med & 51.658 & 50.807 & 1 \\
\hline 2 & Lancet & 39.060 & 36.427 & 1 \\
\hline 3 & JAMA & 29.978 & 29.273 & 1 \\
\hline 6 & Ann Intern Med & 13.976 & 16.260 & 1 \\
\hline 65 & Med Clin Barcelona & 1.399 & 1.254 & 2 \\
\hline 200 & Medicina Buenos Aires & 0.423 & 0.417 & 4 \\
\hline 202 & Rev Med Chile & 0.360 & 0.416 & 4 \\
\hline 206 & Rev Invest Clin México & 0.310 & 0.436 & 4 \\
\hline 222 & Gaceta Med México & 0.128 & 0.172 & 4 \\
\hline
\end{tabular}

*Fuente: Journal of Citation Reports 2012 Published by Thomson Reuters-Medicine: General and Internal (10). "IF" = "Journal Impact Factor"; "5-year IF" = Factor de Impacto promedio de los últimos 5 años; "Q" = Cuartil de ubicación en la categoría analizada. Por su uso habitual en la literatura se han expresado el "IF" y el "5-year IF" separando los decimales con un punto. 
calidad de las publicaciones, destacándose la base de datos Scopus, de la empresa editora Elsevier, que ofrece indicadores de revistas científicas en el portal de libre acceso SCImago Country \& Journal Rank $^{11}$. En noviembre de 2012 se hizo público un documento elaborado por un grupo de estudio de SCImago (SCImago Research Group), encargado por CONICYT-Programa de Información Científica como evaluador externo para analizar la actividad científica chilena, reflejada en publicaciones incluidas en la base de datos Scopus ${ }^{12}$. El estudio comprendió el septenio 2003-2010 e incluyó comparaciones de Chile con algunos países y con la media del mundo. La base de datos Scopus tiene una cobertura superior a $J C R$, ya que incluye a cerca de 19.800 revistas de todas las áreas científicas y humanistas, mientras JCR incluye a 12.300 revistas. A noviembre de 2012 Scopus indizaba 72 revistas chilenas y JCR 48.

El indicador de prestigio SCImago Journal \& Country Rank (SJR) mide la influencia científica de un artículo promedio en una revista y expresa cuán central es para la discusión científica mundial, cualificando el origen de la cita. La Revista Médica de Chile registró una producción de 204 documentos en el año 1999 y 248 en el año 2011. En la categoría "Medicine (miscellaneous)" Scopus incluye 1.664 revistas y su orden de precedencia, según el indicador $S J R$, muestra diferencias notables con respecto a la categoría equivalente en el JCR de ISI-Thomson Reuters. Por ejemplo, en los cuartiles superiores del SJR aparecen revistas no mencionadas en el JCR, algunas de ellas dedicadas a publicar "mini reviews", ocupando posiciones más altas aún que el New England Journal of Medicine. En la clasificación según SJR, la Revista Médica de Chile y la Revista Chilena de Infectología se ubicaron en el cuartil $2(\mathrm{Q} 2)$ y otras 8 revistas chilenas del área de salud estuvieron en el Q4.

Durante los cincuenta años de uso de distintos índices bibliométricos, ellos han sido analizados y criticados porque, muy particularmente el "IF" de ISI-Thomson Reuters, traspasan el prestigio de las revistas a cada uno de los manuscritos publicados en ellas, sin discriminar la importancia individual de cada manuscrito. Además, se ha hecho evidente que el uso del "IF" estimula un sesgo negativo hacia las publicaciones en idiomas no-inglés, aunque aparezcan en revistas de corriente principal ${ }^{6,7}$.

En diciembre de 2012, durante la reunión anual de la American Society for Cell Biology, se proclamó la San Francisco Declaration on Research Assessment, un documento que contiene recomendaciones para mejorar la forma de evaluar la calidad y el impacto de la producción científica ${ }^{13}$. Su primera recomendación es que para calificar las publicaciones de los científicos o para tomar decisiones al contratarlos, promoverlos o financiar sus proyectos de investigación, no se utilicen métricas basadas en el prestigio de las revistas -tales como el "IF"- como sustitutos para medir la calidad individual de sus publicaciones.

\section{Importancia de la difusión electrónica de las revistas, en internet}

La publicación de una revista "on line", sea como forma exclusiva o sumada a su versión impresa, aumenta su visibilidad al facilitar el acceso a un ámbito de lectores mayor que el de los suscriptores de la versión impresa. Esto es más notorio si la versión electrónica se incluye en una base de datos de amplia difusión que permita el acceso libre a los artículos, ya sea al título y el resumen en inglés (caso de PubMed) o, además, a su texto completo (caso de SciELO). La Revista Médica de Chile está disponible en ambas bases de datos.

La Tabla 5 muestra el número de visitas a artículos publicados en las revistas médicas chilenas indizadas en SciELO Chile, en el período 2009 a 2012. La Revista Médica de Chile encabeza la lista, como la visitada mayor número de veces. El sistema de registro en SciELO Chile no permite identificar el origen geográfico de las visitas, pero el alto número de visitas recibido por la Revista Médica de Chile induce a suponer que una proporción importante de ellas procedieron del extranjero. Apoyan esta suposición las Cartas al Editor recibidas del extranjero y que aluden a artículos publicados en la Revista: sus autores no son suscriptores y probablemente el artículo que comentaron lo leyeron en la versión electrónica. Debe tenerse en cuenta que algunas revistas médicas chilenas se publican también en una página web propia, lo que puede aumentar más aún las visitas a sus publicaciones.

Nos pareció interesante identificar los manuscritos que despertaron mayor interés en los lectores de su versión electrónica. La Tabla 6 en lista en orden decreciente a los diez artículos publicados durante el año 2012 que tuvieron 
Tabla 5. Número de visitas anuales en SciELO Chile a artículos publicados en revistas médicas chilenas, en los años 2009 a 2012

\begin{tabular}{|c|c|c|c|c|}
\hline \multirow[t]{2}{*}{ Revista } & \multicolumn{4}{|c|}{ Años } \\
\hline & 2009 & 2010 & 2011 & 2012 \\
\hline Rev Med Chile & 2.758 .745 & 3.367 .843 & 3.084 .891 & 2.437 .250 \\
\hline Rev Chil Pediatría & 1.984 .658 & 2.169 .791 & 1.889 .040 & 1.423 .795 \\
\hline Rev Chil Obstet Ginecol & 958.660 & 1.021 .723 & 974.150 & 777.879 \\
\hline Rev Chil Infectología & 863.491 & 975.232 & 931.131 & 835.561 \\
\hline Rev Chil Nutrición & 529.872 & 637.461 & 656.815 & 557.591 \\
\hline Rev Chil Radiología & 484.263 & 607.637 & 618.198 & 531.878 \\
\hline Rev Chil Neuropsiquiatría & 406.001 & 479.260 & 429.717 & 354.313 \\
\hline Rev Chil Cirugía & 320.988 & 408.981 & 403.802 & 430.729 \\
\hline Rev Chil Enf Respiratorias & 283.832 & 309.369 & 300.691 & 274.828 \\
\hline Rev Chil Cardiología & -- & 32.672 & 34.652 & 43.673 \\
\hline Rev Otorrinolaringol y CCC & 130.876 & 182.555 & 200.126 & 222.468 \\
\hline
\end{tabular}

Tabla 6. "Top Ten" en SciElO Chile 2012: Los diez artículos publicados en la Revista Médica de Chile durante el año 2012, con mayor número de visitas en www.scielo.cl hasta julio de 2013

\begin{tabular}{|c|c|}
\hline n de visitas & Título, Autores, Sección, Mes de publicación \\
\hline 12.892 & $\begin{array}{l}\text { Situación epidemiológica actual del VIH/SIDA en Latinoamérica en la primera década del Siglo XXI: análisis } \\
\text { de las diferencias entre países. } \\
\text { Inmaculada Teva, María Paz Bermúdez, María Teresa Ramiro et al. } \\
\text { Artículo de Investigación, enero }\end{array}$ \\
\hline 4.796 & $\begin{array}{l}\text { Revisión del tratamiento dietético-nutricional de la anorexia nerviosa. } \\
\text { Ignacio Jáuregui-Lobera, Patricia Bolaños-Ríos. } \\
\text { Artículo de Revisión, enero }\end{array}$ \\
\hline 3.887 & $\begin{array}{l}\text { Trastornos neurológicos en niños con síndrome de Down. } \\
\text { Beatriz Gaete, Cecilia Mellado, Marta Hernández. } \\
\text { Artículo de Investigación, febrero }\end{array}$ \\
\hline 3.591 & $\begin{array}{l}\text { Factores asociados con el parto prematuro entre } 22 \text { y } 34 \text { semanas en un hospital público de Santiago. } \\
\text { Alfredo Ovalle, Elena Kakarieka, Gustavo Rencoret et al. } \\
\text { Artículo de Investigación, enero }\end{array}$ \\
\hline 3.046 & $\begin{array}{l}\text { Asociación entre tatuajes, perforaciones y conductas de riesgo en adolescentes. } \\
\text { María Laura Cossio, Laura Giesen F, Gabriela Araya. } \\
\text { Artículo de Investigación, febrero }\end{array}$ \\
\hline 2.952 & $\begin{array}{l}\text { Fenotipos clínicos en enfermedad pulmonar obstructiva crónica: ¿volver al futuro? } \\
\text { Rafael Silva O. } \\
\text { Artículo de Revisión, julio }\end{array}$ \\
\hline 2.733 & $\begin{array}{l}\text { Elaboración del informe médico de lesiones. } \\
\text { Pamela Bórquez V. } \\
\text { Artículo de Revisión, julio }\end{array}$ \\
\hline 2.698 & $\begin{array}{l}\text { Factores de riesgo cardiovascular en estudiantes de la Universidad Austral de Chile. } \\
\text { María Adela Martínez S, Ana María Leiva O, Cristina Sotomayor C. et al } \\
\text { Artículo de Investigación, abril }\end{array}$ \\
\hline 2.469 & $\begin{array}{l}\text { Duración de las licencias médicas FONASA por trastornos mentales y del comportamiento. } \\
\text { Gonzalo Miranda H, Sergio Alvarado O, Jay S Kaufman. } \\
\text { Artículo de Investigación, febrero }\end{array}$ \\
\hline 2.415 & $\begin{array}{l}\text { Diseño y validación del instrumento SALUFAM: un instrumento de valoración de la salud familiar con alto } \\
\text { valor predictivo clínico para la atención primaria chilena. } \\
\text { Klaus Puschel, Paula Repetto, María Olga Solar et al. } \\
\text { Artículo de Investigación, abril }\end{array}$ \\
\hline
\end{tabular}


mayor número de visitas, registradas desde su publicación en www.scielo.cl hasta julio de 2013 (es decir, los “Top Ten” en popularidad entre los visitantes). Esta Tabla muestra el título del artículo, sus tres primeros autores, la Sección de la Revista en que aparecieron y el mes de publicación. Llama la atención que siete de los “Top Ten" fueron Artículos de Investigación, dos de Revisión y uno Especial. Tal vez tres de los artículos de esta lista pudieron ser considerados ajenos al ámbito de interés de lectores que sean médicos generales de adultos o especialistas en Medicina Interna y, sin embargo, están entre los más visitados. Además, los dos primeros de la lista son manuscritos procedentes de universidades españolas y se publicaron en idioma español.
Financiamiento de los manuscritos publicados en 2012

En la mayoría de los manuscritos recibidos en la Revista sus autores no indicaron haber recibido un financiamiento específico para ese trabajo. Habría que suponer que estuvieron respaldados por los recursos disponibles en su sitio de origen, para las labores habituales de los autores. La Tabla 7 muestra que sólo 56 "artículos citables" publicados en 2012 (22\% de los publicados ese año) declararon haber recibido un financiamiento específico por instituciones chilenas, entidades privadas nacionales o con representación en el país. La mayoría fueron Artículos de Investigación $(88 \%)$ y 29 de ellos (52\%) fueron financiados por

Tabla 7. Manuscritos publicados en el año 2012 en la Revista Médica de Chile, en que se reconoció financiamiento especial para el trabajo

\begin{tabular}{|c|c|c|c|c|c|}
\hline Institución financiadora & $\begin{array}{c}\text { Artículos } \\
\text { Investigación }\end{array}$ & $\begin{array}{l}\text { Artículos } \\
\text { Revisión }\end{array}$ & $\begin{array}{l}\text { Artículos } \\
\text { Especiales }\end{array}$ & $\begin{array}{l}\text { Casos } \\
\text { Clínicos }\end{array}$ & Total \\
\hline CONICYT - FONDECYT & 19 & 2 & 1 & 1 & 23 \\
\hline CONICYT - FONDEF & 1 & & & & 1 \\
\hline CONICYT - PIA & & 1 & & & 1 \\
\hline CONICYT / MINSAL - FONIS & 4 & & & & 4 \\
\hline Universidad Católica de Chile & 7 & & 1 & & 8 \\
\hline Universidad de Chile & 3 & & & & 3 \\
\hline WELLCOME TRUST & 2 & & & & 2 \\
\hline INNOVA CHILE & 1 & & & & 1 \\
\hline Laboratorio ORPHAN & 1 & & & & 1 \\
\hline MERCK S.A. & 1 & & & & 1 \\
\hline Municipalidad de Puente Alto & 1 & & & & 1 \\
\hline PFIZER & 1 & & & & 1 \\
\hline Sociedad Chilena de Endocrinología & 1 & & & & 1 \\
\hline Sociedad Chilena de Pediatría & 1 & & & & 1 \\
\hline SODEXO Selecciones de Motivación & 1 & & & & 1 \\
\hline Superintendencia Seguridad Social & 1 & & & & 1 \\
\hline Universidad Austral de Chile & 1 & & & & 1 \\
\hline Universidad de La Frontera & & & & 1 & 1 \\
\hline Universidad de Los Andes & 1 & & & & 1 \\
\hline Universidad de Los Lagos - Osorno & 1 & & & & 1 \\
\hline Universidad de Valparaíso & 1 & & & & 1 \\
\hline Total & 49 & 3 & 2 & 2 & 56 \\
\hline
\end{tabular}


Programas de CONICYT o su asociación con el Ministerio de Salud (FONIS).

Al elegir la Revista Médica de Chile para publicar estos manuscritos, es probable que sus autores consideraran la importancia de difundir su trabajo ante una "audiencia" predominantemente nacional. Cabe citar lo expresado por una académica española ${ }^{6}$ : "...la mejor revista para publicar un trabajo depende de los lectores a los que está dirigido ese trabajo, y esto es más importante que cualquiera obsesión por los factores de impacto" (traducción libre del original en inglés).

\section{Publicación de manuscritos con interés local}

Algunos artículos publicados en la Revista tenían a priori una baja potencialidad de ser citados en revistas extranjeras, porque analizaron temas o situaciones con interés reducido al medio nacional. Esas publicaciones harán crecer el denominador de la ecuación para el cálculo del "IF", sin aumentar su numerador. Sin embargo, los editores consideraron justificada su publicación, coincidiendo -en general- con la opinión de los revisores externos.

\section{¿Debe seleccionarse el contenido de esta Revista guiándose por los índices bibliométricos?}

Nos parece que la respuesta debe ser negativa. Si la Revista Médica de Chile centrara su interés en mejorar los índices bibliométricos dejaría de cumplir con una misión fundacional que la hizo responsable de difundir las experiencias clínicas de los médicos chilenos, los estudios epidemiológicos y descriptivos que caracterizan a nuestros problemas de salud, la tecnología incorporada en el país para el diagnóstico y tratamiento de las enfermedades, las características sociológicas y psicológicas de la población nacional que repercuten en la salud pública y los progresos locales en educación médica, que son elementos con interés fundamentalmente local y regional. Es pertinente resaltar que existen diferencias notorias en la misión de las revistas dedicadas a la medicina clínica y las revistas puramente científicas.

En un artículo atingente a esta materia, Podolsky et al citaron una aseveración interesante: "A la Escuela de Medicina se asiste, de regla, solamente una vez en la vida" ". Cuando J H Salisbury lo dijo, en 1906, no imaginó que la educación médica de post título iba a alcanzar el grado de eficiencia que hoy tiene, en lo cual contribuyen las revistas médicas que son una herramienta crucial para el progreso de los médicos.

Agradecimientos: a la Sra. Marcela Aguirre C., del Programa de Información Científica de SciELO Chile, por su eficiente ayuda en la actualización de datos bibliométricos.

\section{Referencias}

1. Reyes H, Palma J, Andresen M. El manejo editorial de los manuscritos recibidos en la Revista Médica de Chile (Editorial). Rev Med Chile 2004; 132: 7-10.

2. Reyes H, Andresen M, Palma J. ¿Por qué demoran en publicarse los manuscritos en la Revista Médica de Chile? (Editorial). Rev Med Chile 2006; 134: 7-11.

3. Reyes H, Andresen M, Palma J. La Revista Médica de Chile en el año 2009 (Artículo Especial). Rev Med Chile 2009; 137: 1089-94.

4. Garfield E. How can impact factors be improved? BMJ 1996; 313: 411-3.

5. Reyes H. El "factor de impacto" y el impacto de las revistas médicas (Editorial). Rev Med Chile 1998; 126: 135-8.

6. Bordons M. Towards International Recognition of Spanish Scientific Publications (Editorial). Rev Esp Cardiol 2004; 57 (9): 799-802.

7. Jiménez-Contreras E, Torres-Salinas D, Ruiz-Pérez R, Delgado López-Cózar E. Investigación de excelencia en España: ¿protagonistas o papeles secundarios? (Artículo Especial). Med Clin (Barc) 2010: 134 (2): 76-81.

8. Baethge C. Impact Factor - a Useful Tool, but Not for All Purposes. Dtsch Arztebl Int 2012; 109 (15): 267-9

9. Lee SS. Journal's impact and pecking order: tyranny and tyrannosaurs (Editorial). Liver Internat 2012; 32: 1463-4. DOI: $10.1111 /$ liv. 120002

10. ISI Web of Science- Journal Citation Reports, 2012 JCR Science Edition [http://admin-apps.webofknowledge. com/JCR/]

11. SCImago Journal \& Country Rank, [en http://www. scimagojr.com/journalsearch.php?]

12. CONICYT (2012). Principales indicadores bibliométricos de la actividad científica chilena 2010. Informe 2012. Santiago: CONICYT. 228 pág. [Disponible en: http:// www.cincel.cl/Documentos/Recursos/Principales_indicadores_bibliometricos_actividad_cientifica_chilena_Informe_2012.pdf].

13. San Francisco Declaration on Research Assessment. Putting science into the assessment of research. [Disponible en http://am.ascb.org/dora/].

14. Podolsky SH, Greene JA, Jones DS. The Evolving Roles of the Medical Journal (Perspective). N Engl J Med 2012; 366 (16): 1457-61. 